\title{
Phosphate Precipitates and Water-Soluble Aggregates in Re-analyzed Solubility-pH Data of Twenty-five Basic Drugs
}

\author{
Alex Avdeef \\ in-ADME Research, 1732 First Avenue, \#102, New York, NY 10128, USA \\ E-mail:alex@in-ADME.com; Tel.: +1 6466785713 \\ Received: February 15, 2014; Revised: March 12, 2014; Published: April 01, 2014
}

\begin{abstract}
The purpose of the study was to assess the stoichiometries of phosphate precipitates and determine the intrinsic solubilities, $\mathrm{S}_{0}$, of 25 basic drugs from their published solubility-pH profiles in the landmark study of Bergström et al. (2004), where $0.15 \mathrm{M}$ phosphate buffer media had been used. A secondary purpose of this study was to attempt to predict phosphate 1:1 and 2:1 solubility products, $K_{s p}$, from knowledge of $S_{0}$. The published data have been re-analyzed using a novel solubility-pH analysis computer program, pDISOL- $X^{T M}$. The program internally derives implicit solubility equations, given a set of proposed equilibria and constants (which are then iteratively refined by weighted nonlinear regression), and does not require explicit Henderson-Hasselbalch equations. The data were tested for the presence of phosphate precipitates of various stoichiometries, as well as the simultaneous presence of aggregated species, either cationic or neutral. The presence of particular species was suggested by the slope characteristics of the log $S$ vs. $\mathrm{pH}$ curves. Considerably different intrinsic solubility constants were found, compared to those originally reported, for several drugs (e.g., celiprolol, desipramine, haloperidol). The least soluble molecule, amiodarone, analyzed to have the extraordinarily low intrinsic solubility of 2 picograms $/ \mathrm{mL}$, a moderate salt solubility of $0.82 \mathrm{mg} / \mathrm{mL}$ at the Gibbs-p $K_{a} 5.4$, corresponding to the species $B H \cdot H_{2} P_{4}(s)$, and a substantial presence of the positively-charged pentameric aggregate, $(\mathrm{BH})_{5}$.
\end{abstract}

\section{Keywords}

Sparingly-soluble drugs; $\mathrm{pH}$-dependent solubility; salt solubility products; prediction of solubility products; solubility equations; aggregation; miniaturized shake-flask method.

\section{Introduction}

When sparingly soluble basic drugs are added in substantial amount to phosphate-containing buffer solutions, there is likelihood that some drugs will precipitate as phosphate salts in neutral or acidic solutions. Because the buffer can exist in three negatively charged overlapping forms in solution, different phosphate precipitates can form with positively charged ionizable drugs. The process is influenced by the solution $\mathrm{pH}$ in relation to the $\mathrm{pK}_{\mathrm{a}}$ of the drug. If an appreciable amount of chloride is also present in solution, drug-chloride precipitates may form in lieu of, or along with phosphate precipitates. Furthermore, in parallel to precipitation, formation of water-soluble oligomeric drug and micelle species 
can substantially complicate the interpretation of the solubility measurements, especially when the drugs have more than one $\mathrm{p} K_{\mathrm{a}}$.

Although such systems had been studied on a specific case-by-case basis, general computational approaches suitable to tackle the above complexity had been scarce. Perhaps as a result, the need for such analyses had not been widely appreciated. Yet, quantitative analysis of such complex reactions clearly would have beneficial impact in early drug development in terms of clearer interpretations of mechanistic dissolution studies involving salts of sparingly soluble drugs in complex media with which the drugs can directly interact (e.g., such as simulated intestinal fluids) [1-5]. Since salt solubility is a conditional constant, depending on the drug and the specific counterion concentrations, the determination of the thermodynamic solubility product allows for scalability of salt effects across a wide range of concentrations of substituents in formulation research. In addition, the formation of drug aggregates can have significant impact on measured in vitro permeability, since aqueous diffusivity of compounds depends on their size, e.g., as was suggested in the Caco-2 study of retinoic acid in neutral solution [6].

There are numerous reports of aggregation forming systems [1,5-12]. For example, doxycycline [8], with intrinsic solubility $S_{0} 0.72 \mathrm{mg} \cdot \mathrm{mL}^{-1}$, at $25^{\circ} \mathrm{C}$ in $1 \mathrm{M} \mathrm{NaCl} / \mathrm{HCl}$ acidic solutions, forms dimers with $\mathrm{K}_{2}=24 \mathrm{M}^{-1}$. Such aggregates were found to have unusually high solubility, sensitively dependent on temperature. Zhu and Streng [9] found that the self-association of dolasetron to form cationic dimers and trimers was enthalpy driven ( $\mathrm{H}$-bond/aromatic ring rather than hydrophobic/electrostatic interactions), with the aggregation constants, $\mathrm{K}_{2-3}$, ranging from 4 to $50 \mathrm{M}^{-1}$ at $25^{\circ} \mathrm{C}$. Many nonsteroidal antiinflammatory drugs [10], such as indomethacin, diclofenac, ibuprofen, ketoprofen, naproxen, and sulindac, can self-associate by forming mixed-charge micelle or micelle-like structures. The reported CMC of diclofenac is about $25 \mathrm{mM}$ and of ketoprofen is about $160 \mathrm{mM}$ [10]. The re-analysis of the data of Higuchi et al. [7] indicated that barbital and phenobarbital likely formed anionic dimers (reaching peak concentrations near $\mathrm{pH}$ 8), whereas oxytetracycline showed a pronounced tendency to form a cationic dimer (peaking near pH 2) [11]. Saturated alkaline solutions of diprenorphine $(\mathrm{pH}>9)$ appeared to suggest the formation of a mixedcharge anionic dimer [12]. In these and many other similar studies, it is evident that the solubility-pH profiles cannot be described accurately with the Henderson-Hasselbalch equation when aggregates form $[1,5,11-13]$.

This manuscript is the third contribution in a series of studies applying a new salt solubility analysis, refinement, and simulation computer program, $p D I S O L-X^{T M}$ (in-ADME Research), to the study of nonHenderson-Hasselbalch behaving solubility systems [11,12]. The main objective of this brief commentary is to assess the stoichiometries of phosphate precipitates and determine the intrinsic solubilities, $S_{0}$, of 25 basic drugs from their published solubility-pH profiles in the landmark study of Bergström et al. [13], where $0.15 \mathrm{M}$ phosphate buffer media had been used. A parallel purpose of the study was to attempt to predict the 1:1 and 2:1 phosphate solubility products, $K_{\mathrm{sp}}$, from knowledge of $S_{0}$, something that had not been done hitherto, as far as we know.

\section{Experimental}

\section{Legacy Data}

In the Bergström et al. [13] study, the solubility experiments were performed at $23{ }^{\circ} \mathrm{C}$, using a miniaturized shake flask method. Each drug was added in excess to 0.05-0.2 $\mathrm{mL}$ of $0.15 \mathrm{M}$ phosphate buffer. The $\mathrm{pH}$ of each drug suspension was then adjusted using solutions of $\mathrm{H}_{3} \mathrm{PO}_{4}$ or $\mathrm{KOH}$ to a selected $\mathrm{pH}$ (in some cases as low as 1.5 and as high as 12). The suspensions were then agitated at $300 \mathrm{rpm}$ on a 
plate shaker for $24 \mathrm{~h}$, the assigned equilibration time. After $24 \mathrm{~h}$, the samples were centrifuged to separate the precipitate from the solution. The drug concentration in the supernatant was analyzed by HPLC (UV/fluorescence detection). Light scattering measurements did not find colloidal particulates or agglomerates in the supernatant. Practically insoluble amiodarone, carvedilol and mifepristone could not be detected directly by HPLC. Subsequently, these drugs were studied in $6-24 \% \mathrm{w} / \mathrm{w}$ methanol-water mixtures. The corresponding intrinsic solubilities, $S_{0}$, were determined by extrapolation of the watercosolvent solubilities to zero cosolvent. In most cases, the value of $S_{0}$ was assigned to the drug solubility at $\mathrm{pH} \approx \mathrm{p} K_{\mathrm{a}}+2$, where the drug was expected to be predominantly uncharged. The assays appeared to be well designed. However, it would have been helpful presently to have access to the actual amounts of drug added per $\mathrm{mL}$ of buffer in the original study. In addition, it is plausible that some of the practically insoluble drugs had not reached true thermodynamic equilibrium in $24 \mathrm{~h}$. This could be a topic of further study.

\section{Refinement of Intrinsic and Salt Solubility and Aggregation Constants}

The new data analysis method uses log $S-\mathrm{pH}$ as measured input data (along with the standard deviations in log $S$ ) into the $p$ DISOL-X computer program, as described previously $[11,12]$. Solubility values from literature sources are converted to molarity units for processing by the program. Briefly, an algorithm was developed which considers the contributions of all species present in solution, including all components of buffers or mixtures thereof. The approach does not depend on any explicitly derived extensions of the Henderson-Hasselbalch equations [1]. The uniqueness of the computational algorithm is that it derives its own implicit equations internally, given any practical number of equilibria and estimated constants, which are subsequently refined by weighted nonlinear least-squares regression. Drug-salt precipitates, -aggregates, -complexes, - bile salt and -surfactant species can be modeled [1,5]. The presence of specific buffer-drug formed species can be tested. The computer program calculates the distribution of species corresponding to a sequence of additions of standardized strong-acid titrant $\mathrm{HCl}$ (or weak-acid titrants, e.g., $\mathrm{H}_{3} \mathrm{PO}_{4}$ ) to simulate the speciation in the suspension down to $\mathrm{pH} \sim 0$. Subsequently, a sequence of perturbations with standardized $\mathrm{NaOH}$ (or, e.g., $\mathrm{KOH}$ ) is simulated, and solubility calculated at each point (in $\mathrm{pH}$ steps of 0.005-0.2), up to $\mathrm{pH} \sim 13$. The ionic strength is rigorously calculated at each step, and $\mathrm{p} K_{\mathrm{a}}$ values (as well as solubility products, aggregation constants) are accordingly adjusted [11]. At the end of the speciation simulation, the calculated $\log S \mathrm{vs}$. $\mathrm{pH}$ curve is compared to actual measured $\log S$ vs. $\mathrm{pH}$. A log $S$-weighted nonlinear least squares procedure refines the proposed equilibrium model, using analytical expressions for the differential equations. The process is repeated until the differences between calculated and measured $\log S$ values reach a minimum, as described elsewhere in detail [11].

The analysis of the Bergström log $\mathrm{S}-\mathrm{pH}$ data tested for the presence of solid species $\mathrm{B}(\mathrm{s}), \mathrm{BH} \cdot \mathrm{Cl}(\mathrm{s})$, $\mathrm{BHB} \cdot \mathrm{Cl}(\mathrm{s}), \mathrm{BH} \cdot \mathrm{H}_{2} \mathrm{PO}_{4}(\mathrm{~s}),(\mathrm{BH})_{2} \cdot \mathrm{HPO}_{4}(\mathrm{~s}), \mathrm{BHB} \cdot \mathrm{H}_{2} \mathrm{PO}_{4}(\mathrm{~s}),(\mathrm{BHB})_{2} \cdot \mathrm{HPO}_{4}(\mathrm{~s}),(\mathrm{BH})_{3} \cdot \mathrm{H}_{2} \mathrm{PO}_{4} \cdot \mathrm{HPO}_{4}(\mathrm{~s})$, as well as water soluble cationic aggregates $(\mathrm{BH})_{n}$ and $(\mathrm{BHB})_{n}, n=1-6$, and the uncharged aggregate $B_{2}$. Up to three different solids could be tested in a single calculation. The presence of particular species was suggested by the slope characteristics of the log $S$ - $\mathrm{pH}$ curve $[1,5,7-11]$. 


\section{Results and Discussion}

Based on the results of the analysis, the 25 drugs were divided into six groups, according to the type of deviations from the Henderson-Hasselbalch equation in the non-salt regions $\left(\mathrm{pH}>\mathrm{p} K_{\mathrm{a}}{ }^{\mathrm{GIBBS}}[14,15]\right)$ of the $\log S-\mathrm{pH}$ curves, as discussed at length by Avdeef [1].

- CASE 0 (24\% of all drugs): follow Henderson-Hasselbalch equation, and apparent $S_{0}=$ true $S_{0}$.

- CASE 1b (24\%): apparent $\mathrm{p} K_{\mathrm{a}}<$ true $\mathrm{p} K_{\mathrm{a}}$ and apparent $S_{0}>$ true $S_{0}$, which is consistent with the formation of water-soluble uncharged aggregates; degree of aggregation cannot be determined from $\mathrm{pH}$-dependence.

- CASE 2b (16\%): diagonal region of the log $S-\mathrm{pH}$ profile has non-unit slope, indicative of cationic aggregates, where the average degree of aggregation defined by the value of the slope; apparent $\mathrm{S}_{0}=$ true $\mathrm{S}_{0}$.

- CASE 3b (16 \%): diagonal region of the log $\mathrm{S}-\mathrm{pH}$ profile (slope $=-1$ ) shifted to higher $\mathrm{pH}$ than predicted by Henderson-Hasselbalch equation (i.e., apparent $\mathrm{p} K_{\mathrm{a}}>$ true $\mathrm{p} K_{\mathrm{a}}$ ), indicative of mixed-charge dimeric aggregates, $\mathrm{BHB}^{+}$, or higher order oligomers of the same stoichiometric ratio; apparent $S_{0}=$ true $S_{0}$.

- Two group of compounds showed combined CASE $1 b+2 b(8 \%)$ and CASE $2 b+3 b(12 \%)$.

The results of the analysis of the Bergström et al. data are summarized in Table 1, with all constants presented in molarity or molarity related units. Figure 1 compares intrinsic solubility, $p S_{0}\left(-\log _{10} S_{0}\right)$, of the compounds determined in this study to that reported by Bergström et al. [13]. Amiodarone is not shown in Figure 1, since the previously reported value was stated conditionally (Table 1). The eight compounds in Figure 1 represented as checkered circles are all examples of CASE $\mathbf{1 b}$, and showed the largest variances from the reported intrinsic solubility values. On the other hand, the agreement between the filled circle compounds (corresponding to other cases) determined here and reported previously is very good: $p \mathrm{~S}_{0}^{\text {Bergström }}=0.02+1.007 p \mathrm{~S}_{0}{ }^{p \text { DISOL-X }}, \mathrm{r}^{2}=0.99, \mathrm{~s}=0.17, \mathrm{~F}=1014, \mathrm{n}=16$.

Table 1. Summary of the Results of the Re-Analysis of the Bergstrom et al. [13] Data.

\begin{tabular}{|c|c|c|c|c|c|c|c|c|c|c|c|}
\hline COMPOUND & $\begin{array}{l}\mathrm{pK}_{\mathrm{a}}\left(25^{\circ} \mathrm{C}\right. \\
\mathrm{I}=0.15 \mathrm{M})\end{array}$ & $\begin{array}{l}-\log S_{0} \\
\pm S D\end{array}$ & \begin{tabular}{|l} 
Lit. $^{b}$ \\
$-\log S_{0}$
\end{tabular} & $\begin{array}{c}\mathrm{pK}_{\mathrm{sp}} \\
\mathrm{BH}^{+} \cdot \mathrm{H}_{2} \mathrm{PO}_{4}^{-}\end{array}$ & $\begin{array}{c}\mathrm{pK}_{\mathrm{sp}} \\
\left(\mathrm{BH}^{+}\right)_{2} \cdot \mathrm{HPO}_{4}^{2-}\end{array}$ & COMPOUND & $\begin{array}{l}\mathrm{pK}_{\mathrm{a}}\left(25^{\circ} \mathrm{C}, \mathrm{I}\right. \\
=0.15 \mathrm{M})^{\mathrm{a}}\end{array}$ & $\begin{array}{c}-\log S_{0} \\
\pm S D\end{array}$ & \begin{tabular}{|c|} 
Lit. $^{b}$ \\
$-\log S_{0}$
\end{tabular} & $\begin{array}{c}\mathrm{pK}_{\mathrm{sp}} \\
\mathrm{BH}^{+} \cdot \mathrm{H}_{2} \mathrm{PO}_{4}{ }^{-}\end{array}$ & $\begin{array}{c}\mathrm{pK}_{\mathrm{sp}} \\
\left(\mathrm{BH}^{+}\right)_{2} \cdot \mathrm{HPO}_{4}{ }^{2 \cdot}\end{array}$ \\
\hline Amiodarone. $\mathrm{HCl}$ & 10.24 & $11.6 \pm 0.7$ & $7>8.7$ & $7.4 \pm 0.7$ & $15.8 \pm 0.7$ & Maprotiline. $\mathrm{HCl}$ & 10.22 & $4.8 \pm 0.2$ & 4.9 & $3.7 \pm 0.1$ & $7.4 \pm 0.2$ \\
\hline Bupivacaine. $\mathrm{HCl}$ & 8.17 & $3.7 \pm 0.1$ & 3.8 & $2.8 \pm 0.1$ & 5.7 & Mifepristone & 5.23 & $6.0 \pm 0.1$ & 5.9 & 3.8 & 7.9 \\
\hline Carvedilol & 8.06 & $5.9 \pm 0.1$ & 5.9 & $3.8^{\circ}$ & 7.9 & Orphenadrine. $\mathrm{HCl}$ & 9.00 & $3.7 \pm 0.1$ & 4.1 & 2.6 & 5.3 \\
\hline Celiprolol. $\mathrm{HCl}$ & 9.66 & $4.3 \pm 0.2$ & 1.9 & 2.9 & 5.9 & Pramoxine. $\mathrm{HCl}$ & 6.24 & $3.5 \pm 0.1$ & 3.5 & 2.5 & 5.0 \\
\hline Chlorprothixene. $\mathrm{HCl}$ & 8.40 & $6.0 \pm 0.1$ & 5.8 & $4.5 \pm 0.1$ & 9.4 & Procyclidine. $\mathrm{HCl}$ & 10.70 & $5.5 \pm 0.1$ & 4.7 & $2.5 \pm 0.1$ & $4.9 \pm 0.1$ \\
\hline Cyproheptadine. $\mathrm{HCl}$ & 8.87 & $5.7 \pm 0.2$ & 5.9 & $4.1 \pm 0.1$ & $8.8 \pm 0.1$ & Promethazine. $\mathrm{HCl}$ & 9.00 & $5.6 \pm 0.2$ & 5.7 & $4.0 \pm 0.2$ & $8.2 \pm 0.2$ \\
\hline Desipramine. $\mathrm{HCl}$ & 9.28 & $6.4 \pm 0.1$ & 3.9 & $3.0 \pm 0.1$ & 6.1 & Propafenone. $\mathrm{HCl}$ & 9.32 & $5.2 \pm 0.2$ & 5.2 & $3.9 \pm 0.1$ & $7.7 \pm 0.2$ \\
\hline Dipyridamole & 6.17 & $5.1 \pm 0.1$ & 5.5 & $2.6 \pm 0.6$ & 5.3 & Terazosin. $\mathrm{HCl}$ & 7.10 & $3.5 \pm 0.2$ & 1.8 & 2.5 & 5.0 \\
\hline Disopyramide & 10.27 & $3.2 \pm 0.1$ & 3.1 & $2.8 \pm 0.1$ & $5.4 \pm 0.1$ & Thioridazine. $\mathrm{HCl}$ & 9.77 & $6.6 \pm 0.2$ & 4.9 & $4.0 \pm 0.3$ & $8.7 \pm 0.2$ \\
\hline Fendiline. $\mathrm{HCl}$ & 9.00 & $5.3 \pm 0.1$ & 4.0 & $3.3 \pm 0.1$ & 6.8 & Trihexyphenidryl. $\mathrm{HCl}$ & 8.70 & $5.5 \pm 0.1$ & 5.2 & $4.0 \pm 0.2$ & 8.3 \\
\hline Haloperidol & 8.60 & $5.8 \pm 0.1$ & 4.3 & $2.9 \pm 0.3$ & 5.9 & Trimethoprim & 7.14 & $1.9 \pm 0.1$ & 1.9 & $1.3 \pm 0.1$ & $2.9 \pm 0.1$ \\
\hline Hydralazine. $\mathrm{HCl}$ & 7.10 & $2.7 \pm 0.1$ & 2.6 & $5.1 \pm 0.2$ & 10.7 & Verapamil. $\mathrm{HCl}$ & 9.06 & $4.6 \pm 0.1$ & 4.6 & 3.1 & 6.4 \\
\hline Lidocaine & 7.95 & $2.0 \pm 0.1$ & 2.0 & $1.3 \pm 0.1$ & 2.4 & & & & & & \\
\hline
\end{tabular}

${ }^{a} \mathrm{pK}_{\mathrm{a}}$ values taken from the "Wiki-pKa" website: <http://www.in-adme.com/wiki_pka.php/>.

${ }^{b}$ Bergström et al. [13]. ' ${ }^{c}$ Missing constants in red italics were calculated using Eqs. (1) and (2). 


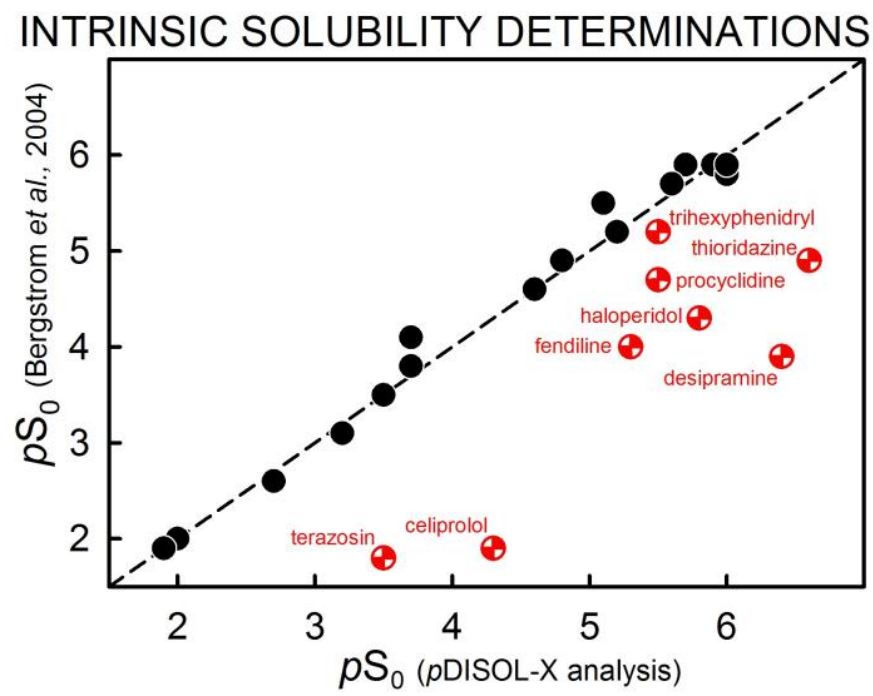

Figure 1. Comparison of previously reported intrinsic solubility values, $p S_{0}$ (molarity based units), to those determined here.

\section{Aggregation Reactions}

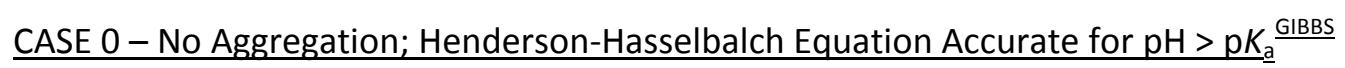

Figure 2 shows the six compounds which show no appreciable deviation from the log $S-\mathrm{pH}$ curves calculated by the Henderson-Hasselbalch equation (non-salt region: $\mathrm{pH}>\mathrm{p} K_{\mathrm{a}}{ }^{\mathrm{GIBBS}}$ ). The compounds are arranged in the order of decreasing intrinsic solubility. Carvedilol and chlorprothixene indicated the lowest intrinsic solubility, with values $<0.5 \mu \mathrm{g} \cdot \mathrm{mL}^{-1}$. The most soluble molecule is lidocaine, with $S_{0}=2.5 \mathrm{mg} \cdot \mathrm{mL}^{-1}$. The $\mathrm{pH}$ at the point of discontinuity between the (most-often flat) salt region and the diagonal slope $=-1$ curve is called $\mathrm{p} K_{\mathrm{a}}{ }^{\mathrm{GIBBS}}[14,15]$. For example, $\mathrm{p} K_{\mathrm{a}}{ }^{\mathrm{GIBBS}}=6.2,9.2,7.0,2.9,6.7,6.0$ for the drugs in Figure 2, in the order of their appearance.

With the exception of verapamil and carvedilol, simple 2:1 and/or 1:1 phosphate salts appear to form with this class of compounds. Verapamil data in the salt region $\left(\mathrm{pH}<\mathrm{p} K_{\mathrm{a}}{ }^{\mathrm{GIBBS}}\right)$ are consistent with the formation of a chloride salt with the mixed-charge dimer of verapamil, $\left(\mathrm{BHB}^{+}\right) \cdot \mathrm{Cl}^{-}(\mathrm{s})$. The region below $\mathrm{pH} 7$ displays a slope $=-0.5$, characteristic of such a stoichiometry. Carvedilol salt region is best fit with a diphosphate stoichiometry: $3 \mathrm{BH}^{+}+\mathrm{H}_{2} \mathrm{PO}_{4}{ }^{-}+\mathrm{HPO}_{4}{ }^{2-} \leftrightarrows(\mathrm{BH})_{3} \mathrm{H}_{3}\left(\mathrm{PO}_{4}\right)_{2}(\mathrm{~s})$.

Verapamil and carvedilol (and mifepristone, below) illustrate why it is better to designate the $\mathrm{pH}$ of discontinuity as $\mathrm{p} K_{\mathrm{a}}^{\mathrm{GIBBS}}$, rather than " $\mathrm{pH}_{\max }$, since the discontinuity can occur at a $\mathrm{pH}$ where the solubility is not at all at its maximum value [14,15]. 

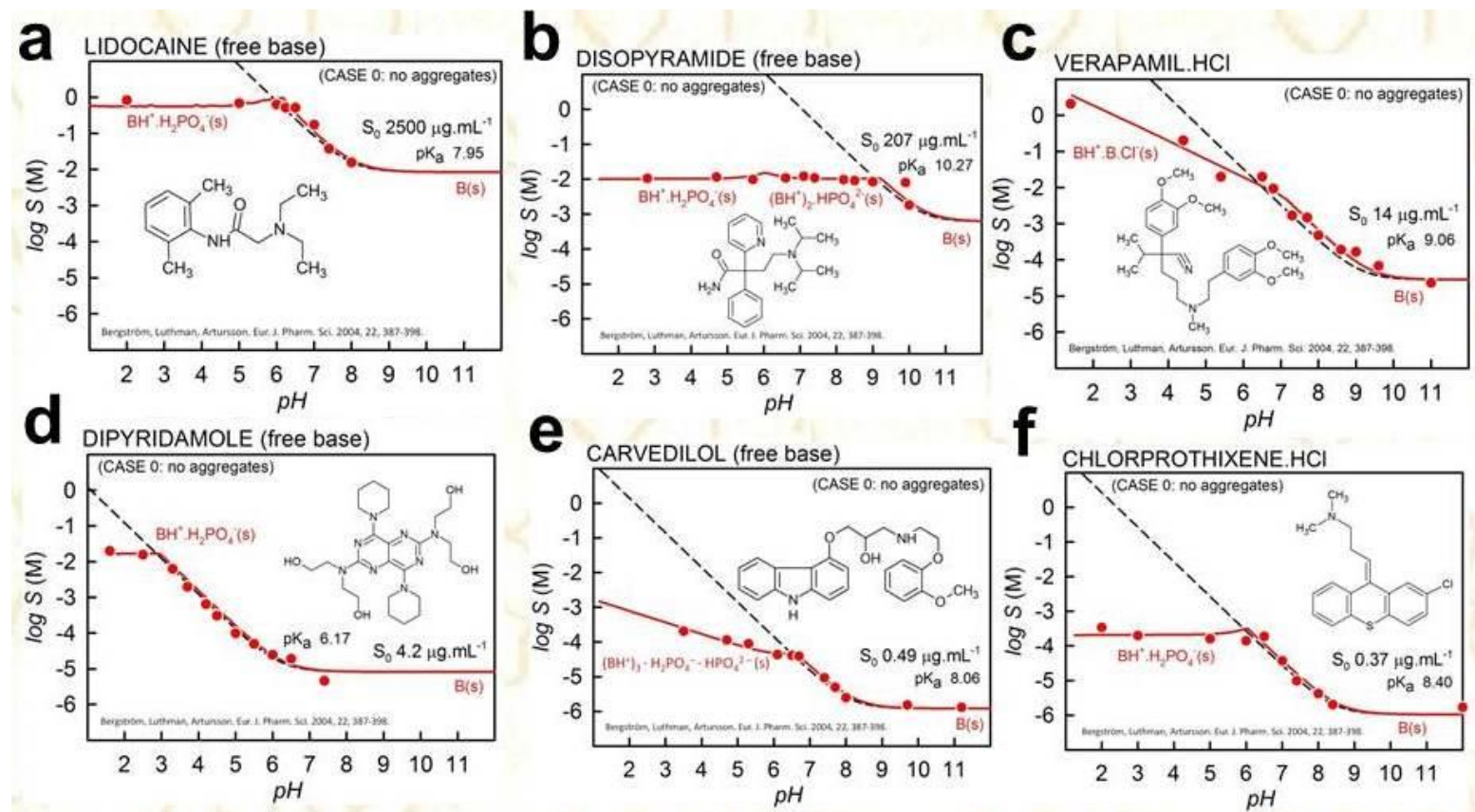

Figure 2. CASE 0 compounds which show Henderson-Hasselbalch form log $S-\mathrm{pH}$ curves in the non-salt region.

\section{CASE 1b - Neutral Aggregates; Henderson-Hasselbalch Equation Not Accurate}

Figure 3 shows the six compounds with apparent $\mathrm{p} K_{\mathrm{a}}<$ true $\mathrm{p} K_{\mathrm{a}}$, which is consistent with the formation of water-soluble uncharged aggregates. The Henderson-Hasselbalch equation does not accurately predict the $\log \mathrm{S}-\mathrm{pH}$ curves across the entire $\mathrm{pH}$ range. For CASE $1 \mathrm{~b}$ drugs, the apparent $S_{0}>$ true $S_{0}$. The degree of aggregation cannot be determined from $\mathrm{pH}$-dependence, since the aggregated compound is in the uncharged form. The compounds in Figure 3 are arranged in the increasing order of aggregation strength.
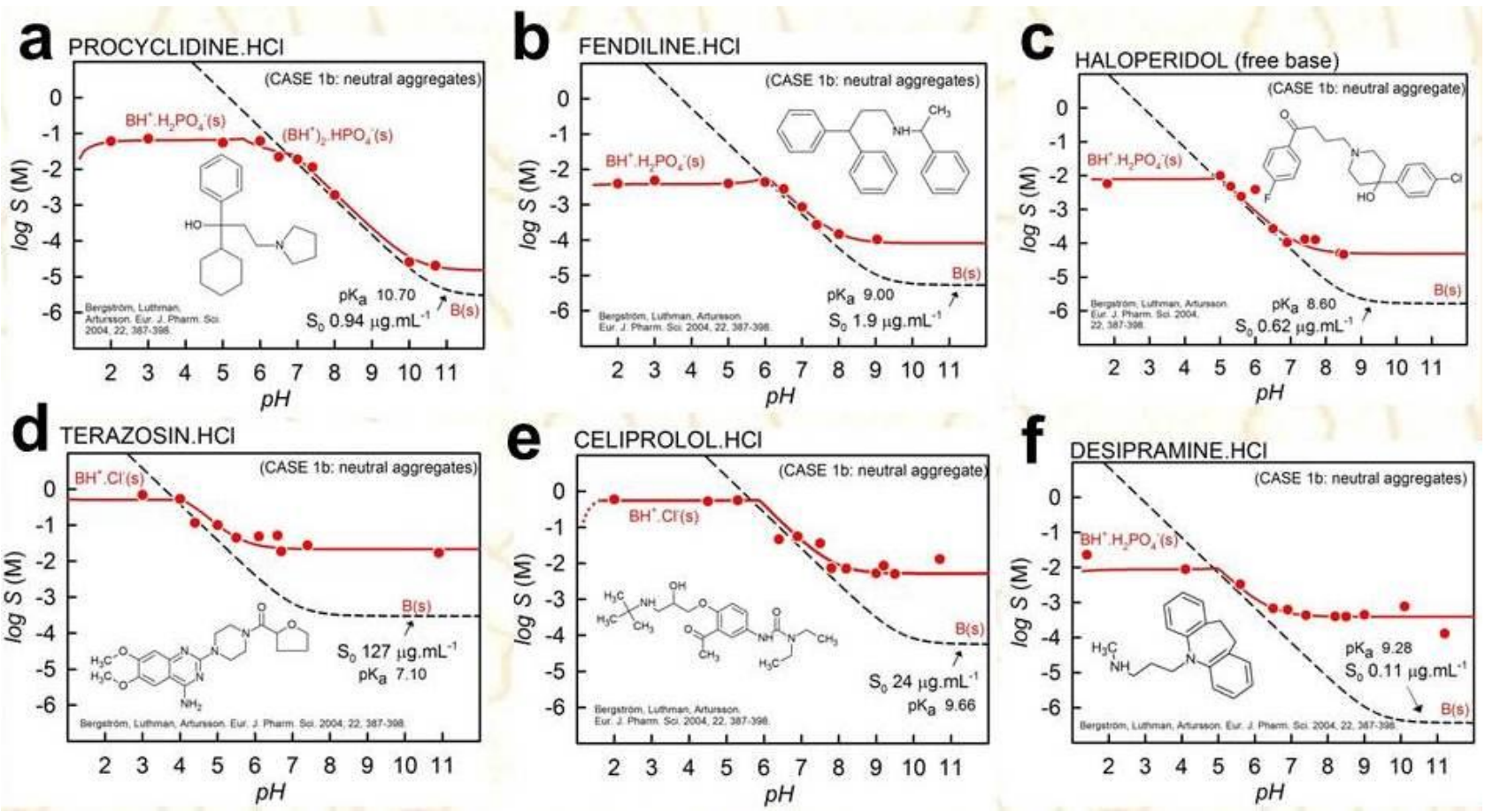

Figure 3. CASE $1 \mathrm{~b}$ compounds: log $S-\mathrm{pH}$ curve distortions consistent with the formation of neutral aggregates. 
The compounds in the Figure 3 either (i) form stable water-soluble aggregates of the uncharged drug, or (ii) the compounds show a pattern of curve distortion, which is characteristic of a system that had not reached a true state of thermodynamic equilibrium at the $24 \mathrm{~h}$ time point. That is, neutral aggregates may be thermodynamically unstable and are very slowly coalescing into solid form. Examples of the former (i) effect have been reported with molecules like piroxicam [1,5], and cases of the latter (ii) effect have been reported for terfenadine, which at $24 \mathrm{~h}$ showed more pronounced distortion that at $68 \mathrm{~h} \mathrm{[1,5]}$ (it would be useful to repeat the measurements of the six drugs in Figure 3 at much longer equilibration times than the $24 \mathrm{~h}$ actually used). It is evident that to analyze CASE $1 \mathrm{~b}$ compounds, it is necessary to know the true $\mathrm{p} K_{\mathrm{a}}$ accurately.

It is with this class of compounds that there were the largest deviations in Figure 1. Apparently, the published intrinsic values [13] ascribed the reported intrinsic solubility to the apparent intrinsic values (the minimum red (solid) curve value rather than the minimum dashed curve value). The six examples in the above figure show flat curves in the salt region, corresponding to simple stoichiometry phosphate or chloride salts.

\section{CASE 2b-Cationic Aggregates; Henderson-Hasselbalch Equation Not Accurate}

Figure 4 shows four compounds which show non-unit slopes in a part of the diagonal region of the $\log \mathrm{S}-\mathrm{pH}$ profile. This is consistent with the formation cationic aggregates. The average degree of aggregation is defined by the value of the slope. Apparent $S_{0}=$ true $S_{0}$.
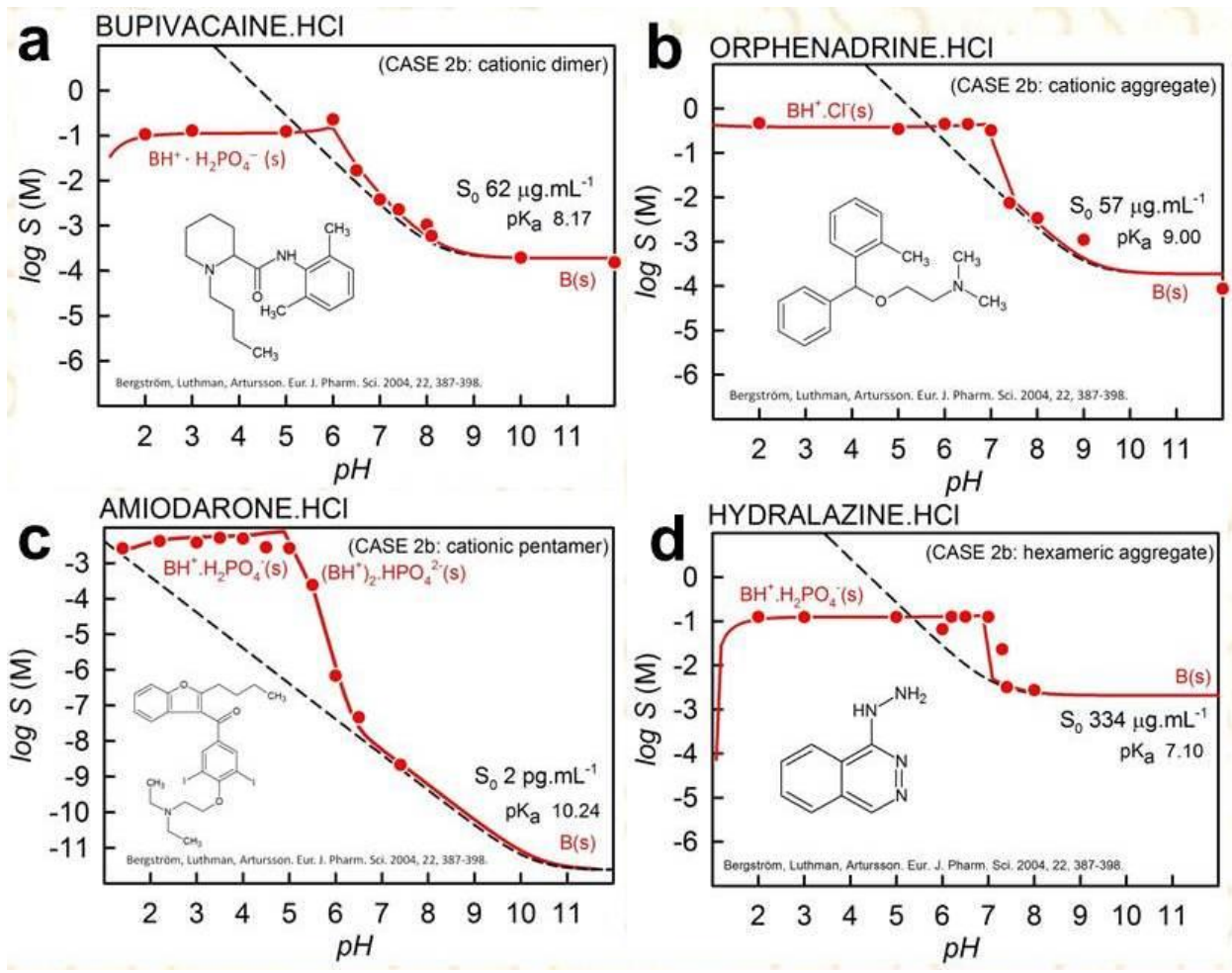

Figure 4. CASE $2 \mathrm{~b}$ compounds which show $\log S-\mathrm{pH}$ curve distortions consistent with the formation of cationic aggregates.

Bupivacaine analyzed as consisting of $\left(\mathrm{BH}^{+}\right)_{2}$ cationic dimers for $\mathrm{pH}<7$, co-existing with the 1:1 phosphate precipitate. This raises the observed solubility in the salt region, due to competition between 
the drug in the solid and aggregated states. Consequently, greater amount of compound added to solution makes the compound more soluble in the salt region. The data shape of the other three molecules was most consistent with the presence of pentameric or hexameric cationic aggregates.

It was possible to rationalize the amiodarone $\log S-\mathrm{pH}$ profile as being distorted from the shape predicted by the Henderson-Hasselbalch equation due to the formation of the pentamer $\left(\mathrm{BH}^{+}\right)_{5}$. If this were a valid interpretation of the data, then there are some very interesting consequences. In the analysis of the amiodarone data, it was assumed that $5 \mathrm{mg}$ of drug were added to $1 \mathrm{~mL}$ of buffer. The $\mathrm{pH}<5$ region of solubility curve (Fig. 4c) depends on both the presence of the cationic aggregate and the phosphate precipitate. Since the concentration of the aggregate depends steeply on the amount of amiodarone added to the buffer, a series of simulations with decreasing amounts of amiodarone results in lowering the solubility in the salt region. In the extreme, maximum solubility can decrease from about $10^{-2}$ (Fig. 4c) to $10^{-7} \mathrm{M}$ (not shown). This suggests that a parenteral injectable solution can show solubility as high as 5 $\mathrm{mg} \cdot \mathrm{mL}^{-1}$ in the $\mathrm{pH}$ 1-5 region, provided enough drug were present in the formulation. However, if a much smaller amount of drug were added, then the solubility could be limited by the apparent minimum $\mathrm{p} K_{\mathrm{sp}, 1: 1}$ $\left(-\log _{10} K_{\mathrm{sp}, 1: 1}\right)$, to about $14 \mathrm{ng} \cdot \mathrm{mL}^{-1}$, with no influence of the aggregate, according to the $p D I S O L-X$ simulation calculation.

Amiodarone is the drug of choice in the treatment of arrhythmia, but it has side effects that limit its long-term use. In the treatment of chronic arrhythmia, oral doses of amiodarone may take 30 days to develop full therapeutic effect because of the extreme hydrophobicity of the drug. Once treatment is discontinued, it may take 1-2 months to wash out the drug completely. On the other hand, during recovery from heart surgery, the atrium can start to fibrillate dangerously. One remedy is to inject an amiodarone parenteral formulation [16] into a vessel carrying blood into the heart. Within a few minutes, the drug can take effect. The analysis of the log $S-\mathrm{pH}$ profile of amiodarone can shed light on the above two seemingly incompatible circumstances.

Hydralazine was problematic to analyze. Figure $4 \mathrm{~d}$ shows a red (solid) curve based on the assumed presence of a hexameric $\left(\mathrm{BH}^{+}\right)_{6}$ species, which is most consistent with the data that deviates most from the dashed line in the non-salt region. However, the consequence of such a species being present in parallel with the phosphate precipitate is that the pKsp,1:1 would analyze as $5.12 \pm 0.24$ (the value in Table 1). Since the $\mathrm{p} S_{0}$ value is unaffected by the cationic aggregate, the relationship between the $K_{\mathrm{sp}}$ and $S_{0}$ can be dependent on the aggregation model. It will be suggested below that it may be more meaningful to assume that the data in the deviation region $\mathrm{pH}$ 6-7 is not at equilibrium. When the data are excluded from the analysis and no stable aggregate is assumed present, then $\mathrm{p} K_{\mathrm{sp}, 1: 1}$ analyzes as $2.74 \pm 0.04$. The latter value, which follows the relationship in Figure 8a more closely, was used in the plot and regression analysis.

\section{CASE 3b - Mixed-Charge Cationic Dimers; Henderson-Hasselbalch Equation Not Accurate}

Figure 5 shows four examples of CASE $3 b \log S-\mathrm{pH}$ profiles. These are characterized by the (slope $=-1$ ) diagonal region of the log $S-\mathrm{pH}$ profile shifted to higher $\mathrm{pH}$ (i.e., apparent $\mathrm{pKa}>$ true $\mathrm{pKa}$ ) than predicted by Henderson-Hasselbalch equation, indicative of mixed-charge dimeric aggregates, $\mathrm{BHB}^{+}$. In such cases, the apparent $S_{0}$ is the same as the true $S_{0}$.

The compounds are arranged in the order of increasing dimerization formation constant, with trimethoprim nearly showing Henderson-Hasselbalch characteristic of CASE 0. Mifepristone appears to 
show a phosphate salt formation stoichiometry $\left(\mathrm{BHB}^{+}\right) \cdot \mathrm{B} \cdot\left(\mathrm{H}_{2} \mathrm{PO}_{4}{ }^{-}\right)(\mathrm{s})$, characterized by an average slope $-0.53 \pm 0.06$ in the $\mathrm{pH} 1.0-3.4$ salt region, similar to the case of verapamil (Fig. 2c). Trimethoprim and promethazine. $\mathrm{HCl}$ show simple 1:1 and 2:1 phosphate salts.
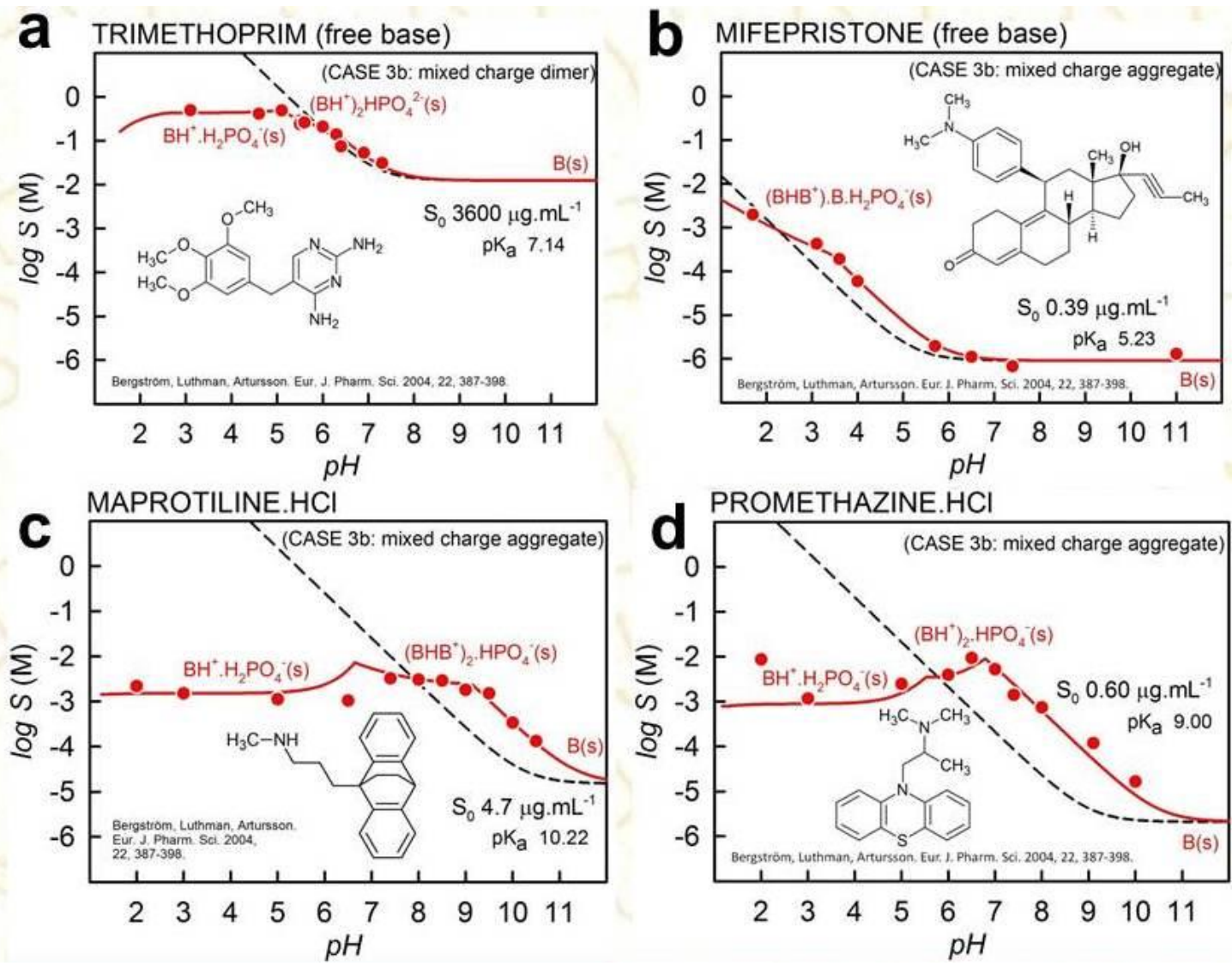

Figure 5. CASE $3 b$ compounds which show $\log S-\mathrm{pH}$ curve distortions consistent with the formation of mixed-charge dimeric aggregates.

Mixtures of Cases; Henderson-Hasselbalch Equation Not Accurate

Figures 6 and 7 show examples of mixtures of CASE $1 b+2 b$ and $2 b+3 b$, respectively. The CASE $1 b$ distortion in thioridazine may be due to incomplete equilibration time.
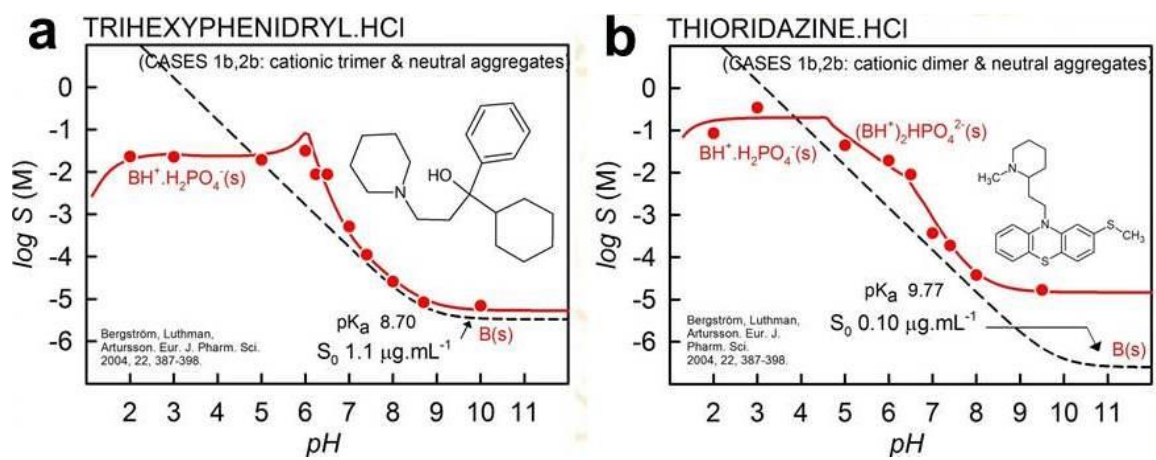

Figure 6. Two examples of compounds showing two overlapping distortion types: CASE $1 \mathrm{~b}$ (neutral aggregates) and CASE $2 b$ (cationic aggregates). 

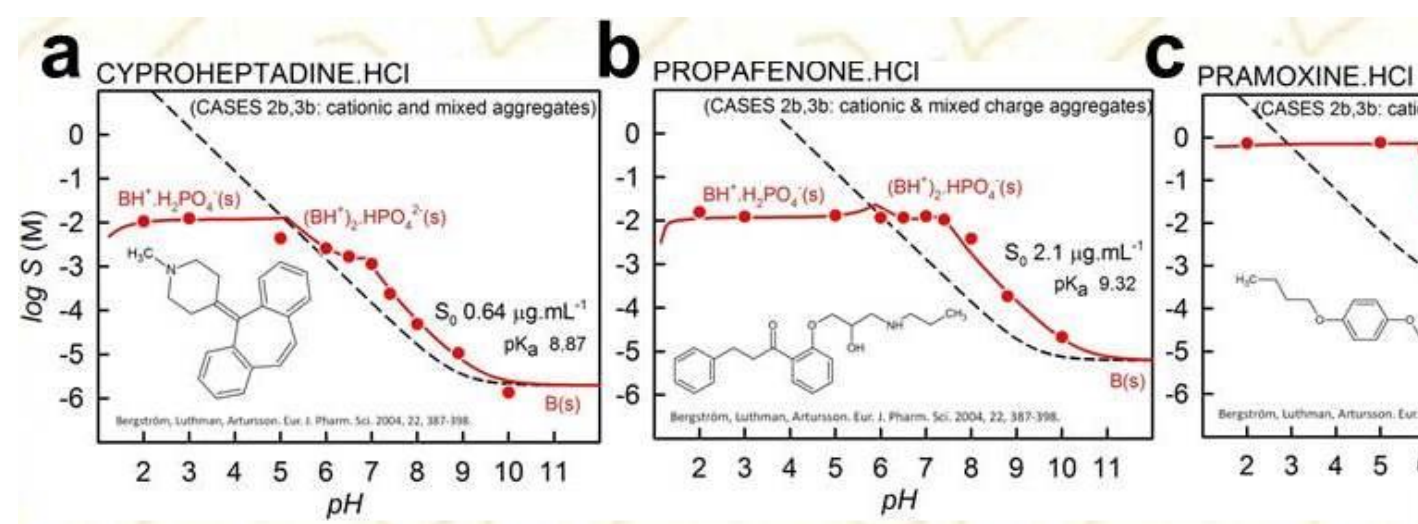

Figure 7. Three examples of compounds showing two overlapping distortion types: CASE $2 b$ (cationic aggregates) and CASE 3b (mixed-charge dimers).

\section{Relationships between Intrinsic Solubility and the Two Phosphate Solubility Products}

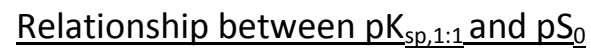

Just 18 of the 25 drugs considered were interpreted to include $1: 1 \mathrm{BH} \cdot \mathrm{H}_{2} \mathrm{PO}_{4}$ salt formations. The other seven drugs were either salts with complicated phosphate stoichiometry or chloride salts (for very soluble drugs introduced as hydrochlorides). The average of the ratio values $p K_{s p, 1: 1} / p S_{0}$ is $0.73 \pm 0.34$. Figure $8 a$ shows the empirical correlation between the $p K_{\mathrm{sp}, 1: 1}$ constant and the intrinsic solubility $p S_{0}$ for the 18 compounds. A trend is evident, in that the less soluble the uncharged form of the drug, the lower is the $K_{\mathrm{sp}}$ value. Although the variance is relatively large, the relationship can still be a valuable basis for prediction of the phosphate solubility product in the absence of measurement.
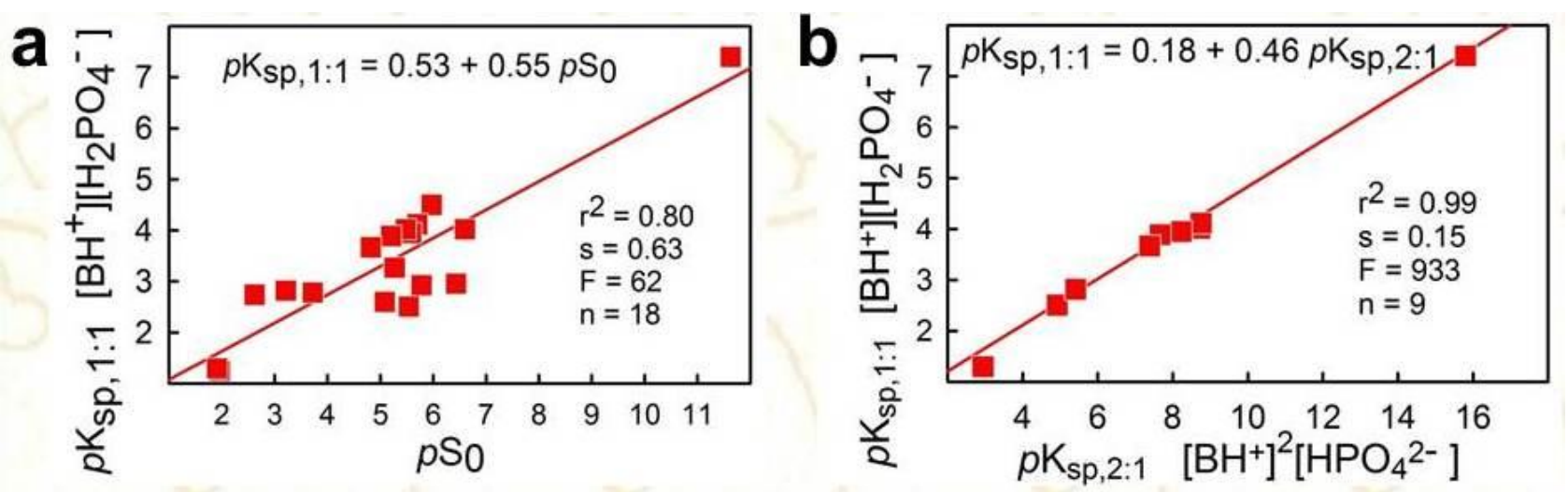

Figure 8. (a) Correlations between the $1: 1$ salt solubility products and the intrinsic solubility constants for 18 of the studied compounds. (b) Correlation between 1:1 and 2:1 drug-phosphate solubility products for 9 of the studied compounds. The bracket concentration products (molarity related units) in the labels have $-\log _{10}$ implied.

Relationship between $\mathrm{p} K_{\underline{s p}, 1: 1}$ and $\mathrm{p} K_{\underline{s p}, 2: 1}$

Nine of the 25 drugs were interpreted to have both 1:1 and 2:1 phosphate salt precipitation. Figure $8 \mathrm{~b}$ shows an extraordinarily high correlation between the two salt solubility products. This is probably expected from equilibrium equation considerations, since the slope of 0.46 is so close to the one-to-two ratio of stoichiometries. The average ratio of the two $\mathrm{p} K_{\mathrm{sp}}$ constants is $0.50 \pm 0.04$ in Figure $8 \mathrm{~b}$. 


\section{Prediction Equations for Phosphate Salts}

From the correlation relationships in Figure 8, two useful empirical equations may be derived:

$$
\begin{aligned}
& \mathrm{p} K_{\mathrm{sp}, 1: 1}=0.525+0.554 p \mathrm{~S}_{0} \\
& \mathrm{p} K_{\mathrm{sp}, 2: 1}=0.744+1.207 p \mathrm{~S}_{0}=-0.4002+2.1796 p K_{\mathrm{sp}, 1: 1}
\end{aligned}
$$

These equations were used to fill in the "missing" values in Table 1, indicated in red italic numbers.

\section{Chloride Salts}

Since some relatively soluble salts were introduced as drug hydrochlorides, the solubility data were tested for the possibility of chloride precipitates. The log $S-\mathrm{pH}$ plots for celiprolol, orphenadrine, terazosin and pramoxine appeared to be consistent with the formation of 1:1 chloride salts. The salt region in the solubility plot is expected to be a horizontal line, since the common ion effect is not expected when the $\mathrm{pH}$ is adjusted using $\mathrm{H}_{3} \mathrm{PO}_{4}$. However, the above assignments are tentative without additional data collected at different added amounts of these drugs, especially with data taken to very low $\mathrm{pH}$ values.

\section{How Constant Are Solubility Products?}

The pDISOL-X program calculates the ionic strength at each $\mathrm{pH}$ point in the processing of the $\log S-\mathrm{pH}$ data, as described in detail elsewhere [11]. Figure 9 shows the speciation analysis of disopyramide in the phosphate salt region, $\mathrm{pH} 2-9$. Figure $9 \mathrm{~b}$ shows that the ionic strength varies from about 0.1 to $0.4 \mathrm{M}$ as the $\mathrm{pH}$ changes from 2 to 9 . The vertical dotted line near $\mathrm{pH} 6$ in Figure 9 a indicates the transition $\mathrm{pH}$ between the 1:1 and the 2:1 phosphate salts. The ionic strength dependence of the two $K_{\text {sp }}$ constants differs, which affects the calculated concentrations. The result of this is that the solubility product above $\mathrm{pH} 6$ is not exactly constant, due to the effect of ionic strength. The analysis program is particularly well suited to explore the consequences of the choices of various species tested in the solubility model. 


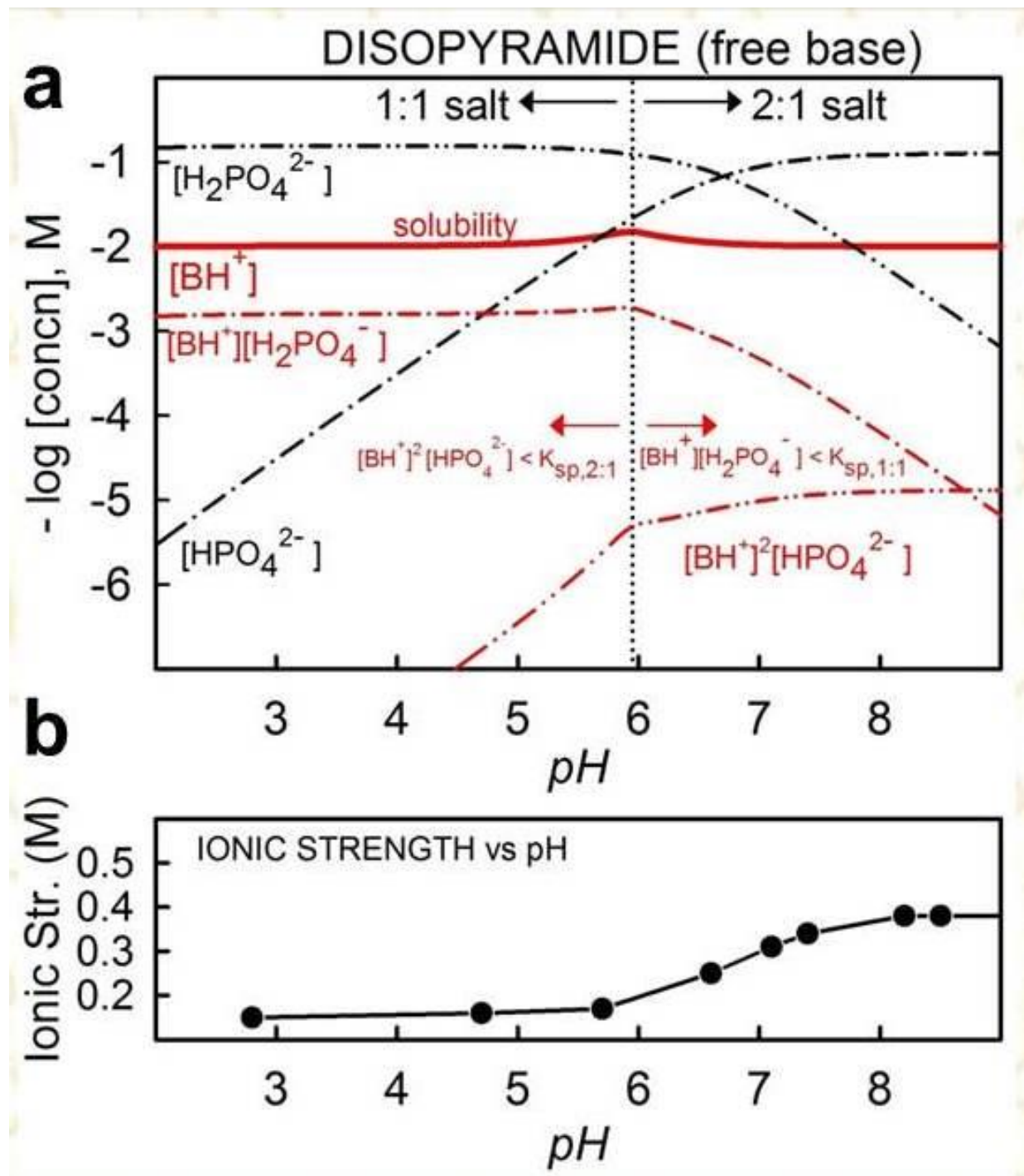

Figure 9. The effect of the ionic strength on the constancy of the solubility product.

\section{Conclusions}

Evidently, concentrated phosphate buffers $(0.15 \mathrm{M})$ can dramatically influence the solubility profiles of sparingly soluble drugs. Clearly, the Bergström et al. [13] data for the 25 compounds studied could not be explained by the simple Henderson-Hasselbalch equation in all but six cases. Apparently, the aggregated species proposed here were either not recognized originally, or were not fully explored. Such anomalies may be common with sparingly soluble drugs, but are not always easy to recognize. $p$ DISOL-X could be a helpful new tool in early development to further aid in the analysis of dissolution mechanisms of sparingly soluble drugs, which depend on the stoichiometry and solubility of drug species, especially of salts.

\section{Acknowledgements}

Helpful discussions with Christel Bergström of Uppsala University, and Gergely Völgyi and Krisztina Takács-Novák of Semmelweis University (Budapest) are greatly appreciated. 


\section{References}

[1] A. Avdeef. Absorption and Drug Development Second Edition, Wiley-Interscience, Hoboken, NJ, 2012, pp. 251-318.

[2] A. Glomme, J. März, J.B. Dressman. In: B. Testa, S.D. Krämer, H. Wunderli-Allenspach, G. Folkers (Eds.), Pharmacokinetic Profiling in Drug Research: Biological, Physicochemical, and Computational Strategies, Wiley-VCH, Weinheim, 2006, pp. 259-280.

[3] J.H. Fagerberg, O. Tsinman, K. Tsinman, N. Sun, A. Avdeef, C.A.S. Bergström. Mol. Pharmaceutics 7, (2010) 1419-1430.

[4] A. Avdeef, O. Tsinman. Pharm. Res. 25 (2008) 2613-2627.

[5] A. Avdeef. Adv. Drug Deliv. Rev. 59 (2007) 568-590.

[6] A. Avdeef, K.Y. Tam. J. Med. Chem. 53 (2010) 3566-3584.

[7] T. Higuchi, M. Gupta, L.W. Busse. J. Am. Pharm. Assoc. 42 (1953) 157-161.

[8] J.B. Bogardus, R.K. Blackwood, Jr. J. Pharm. Sci. 68 (1979) 188-194.

[9] C. Zhu, W.H. Streng. Int. J. Pharm. 130 (1996) 159-168.

[10] A. Fini, G. Fazio, G. Feroci. Int. J. Pharm. 126 (1995) 95-102.

[11] G. Völgyi, A. Marosi, K. Takács-Novák, A. Avdeef. ADMET \& DMPK 1(4) (2013) 48-62.

[12] A. Avdeef. ADMET \& DMPK 2 (2014) 33-42

[13] Bergström CAS, Luthman K, Artursson P. Eur. J. Pharm. Sci. 24 (2004) 387-398.

[14] W.H. Streng. Int. J. Pharm. 186 (1999) 137-140.

[15] A. Avdeef. Pharm. Pharmacol. Commun. 4 (1998) 165-178.

[16] Amiodarone-containing parenteral solution patent: EP 1267865 B1 (15 Feb 2006). 